\title{
La calidad percibida por los participantes en dos ediciones de una carrera popular
}

\author{
Salvador Angosto Sánchez (D), José María López Gullón(D), Arturo Díaz SuáreziD \\ Facultad Ciencias del Deporte. Universidad de Murcia (Spain) \\ salvador.a.s@um.es, luchamurcia@um.es, ardia?@um.es
}

Received April, 2016

Accepted May, 2016

\section{Resumen}

Objeto: La presente investigación se basa en valorar la calidad percibida de los participantes en dos ediciones de una carrera popular de media distancia, examinando las diferentes características que presentan este tipo de eventos.

Diseño/metodología/enfoque: El estudio se ha realizado mediante un cuestionario de calidad percibida para carreras populares desglosándolo en los diferentes factores identificados en estudios previos. Se administró de forma telemática los cuestionarios a los participantes en cada una de las ediciones.

Aportaciones y resultados: Los resultados indicaron un descenso en la valoración de la calidad percibida de la prueba en la siguiente edición donde el factor personal fue el más valorado en ambas ediciones. Las mujeres tuvieron una mejor opinión que los hombres en ambos eventos y en todas las dimensiones.

Originalidad / Valor añadido: El análisis de la calidad percibida desde el punto de vista del participante va a ayudar a los organizadores y empresas deportivas, públicas o privadas, dedicadas a la gestión de eventos deportivos a ser más eficientes y tener una mejor organización.

Palabras clave: Gestión deportiva, Evento deportivo, Calidad percibida, Carrera popular 
Códigos JEL: L83

Title: Participants' perceived quality in two editions of a popular race

\section{Abstract}

Purpose: The present research is based on assessing the participants' perceived quality in two editions of a middle distance popular race, examining the different characteristics these type of events.

Design/methodology: The study was conducted through a perceived quality questionnaire for popular races breaking it down into the different factors identified in previous studies. The questionnaire was administered to participants in each of the editions by email.

Findings: The results of this study indicated a decrease in the valuation of the perceived quality of the second edition, where the personal factor was the most valued in both editions. Women had a better opinion than men in both events and in all dimensions.

Originality/value: The perceived quality analysis from the participants' point of view will help the sports organizers and companies, public or private, engaged in the sporting events management to be more efficient and have better organization.

Keywords: Sport management, Sporting event, Perceived quality, Popular race

Jel Codes: L83

\section{Introducción}

En la actual sociedad se está necesitando cada vez más la necesidad de adquirir tiempo libre y de ocio, con la percepción de que esta adquisición de tiempo libre y de ocio, va en aumento, siendo éste cada vez más utilizado para realizar prácticas de actividad física o deporte, considerándose como una de las prácticas más populares, bien con el disfrute de la práctica en sí o con el disfrute como espectador. 
En cuanto a los grandes eventos existentes, considerados trascendentales para la economía de muchos sitios, generan bastantes beneficios para la economía, es por ello que existen muchísimos intereses por parte de estos lugares que desean ser sede de muchos eventos físicos-deportivos.

En relación a estos grandes eventos o cualquier evento que se realice, de la magnitud que sea, se debe de disponer de una organización y una eficiente gestión, con el fin de lograr una percepción positiva tanto para el deportista como para el espectador, para lo que necesita cuidar aspectos relacionados con la calidad del servicio y la satisfacción de los usuarios que son partícipes de estos eventos. En cuanto a la conceptualización de la calidad, en un principio se contextualizó con relación a un mero producto, siendo más tarde cuando se enfocó hacia el cliente-usuario, finalizando poco a poco en el pensamiento subjetivo de la necesidad del cliente, apareciendo el concepto de calidad percibida.

Shone y Bryan (2010, pp. 4) realizan una definición de los eventos en general como "fenómenos que surgen de ocasiones no rutinarias y que tienen objetivos de ocio, culturales, personales u organizativos establecidos de forma separada a la actividad normal diaria, cuya finalidad es ilustrar, celebrar, entretener o retar la experiencia de un grupo de personas".

En la actualidad dentro del ámbito empresarial, el concepto calidad es un tema crucial e importante. Cada vez más las empresas, tanto a nivel general como en el nivel deportivo, estudian ofrecer unos productos o servicios con la máxima calidad, con un fin común, poder llamar la atención de los futuros clientes o fidelizar los que la empresa tiene, sobre todo en la época que estamos viviendo, ya que es crucial para la supervivencia de la empresa.

Actualmente la calidad es bien necesario y básico que debe de tener la empresa a la hora de la elaboración de estrategias, con un fin principal, que es diferenciarse de las diferentes empresas del mercado, asegurando el progreso y estabilidad en el mercado tan competitivo que existe.

Dentro de los servicios, un aspecto importante que marca la diferencia con respecto a los productos, es que presentan características muy concretas, como la heterogeneidad, intangibilidad e inseparabilidad, ya que se produce y se consume en el momento y no tienen un propietario concreto (Afthinos, Theodorakis \& Nassis, 2005; Chelladurai \& Chang, 2000; Parasuraman, Zeithaml \& Berry, 1985).

El gran interés por el conocimiento de cómo perciben los clientes el grado de calidad de los servicios deportivos, es una de las principales herramientas en las que las organizaciones se apoyan y utilizan como una forma de profundizar en las características de los clientes o usuarios. Por ello, para lograr competitividad y eficiencia, los organizadores se esfuerzan por comprender las necesidades de sus 
clientes y recurren al análisis de la calidad de su servicio y la satisfacción de los usuarios. (Rosa, Castellanos \& Palacios, 2012)

El perfil de la población, sus necesidades y demandas son un tema importante para su conocimiento, siendo determinante para lograr la máxima competitividad (Mármol, Orquín Castrillón \& Sainz de Baranda, 2010) permitiendo un adecuado desarrollo de los planes de calidad, aportando una eficacia y eficiencia en la gestión de organizaciones deportivas (Gálvez, Morales-Sánchez, Hernández-Mendo, Pérez-López \& López-Linares, 2012).

Mundina y Calabuig (2011) piensan que cada vez es más necesario realizar estudios que valoren la conducta del consumidor deportivo, fortaleciendo y manteniendo relaciones con los usuarios en el tiempo, con el objetivo de satisfacerlos y establecer una estrecha relación con ellos, creando un lazo de unión con el evento que se traducirá en éxito para la organización.

Según Calabuig, Molina y Núñez (2012), la calidad del servicio se podría definir como una evaluación a largo plazo post-consumo o post-uso, susceptible de cambio en cada transacción, fuente de desarrollo y cambio de las actitudes hacia el objeto de consumo o uso, y que es el resultado de procesos psicosociales de carácter cognitivo y afectivo.

En la dimensión en referencia a la imagen corporativa de la empresa establecida por Grönroos (1984) como valor de marca y considerándola que según la percepción que tenga el cliente de la empresa, considerando que las personas si tienen una imagen buena, si ocurre cualquier mínimo error se considera subsanable y con una forma puntual, mientras que si tiene una mala percepción cualquier pequeño error es visto con gran magnitud y con una mayor frecuencia de producción del mismo, repercutiendo todo ello en la fidelización y lealtad de los clientes hacia el producto o servicio.

En relación a Clemes, Brush y Collins (2011) en su investigación determina dos corrientes para poder orientar a los gestores, con deseo de mejorar la experiencia de los clientes o usuarios. En la primera de estas corrientes encontramos una investigación de factores que influyen en la satisfacción del cliente, siendo en la segunda, las dimensiones de calidad del servicio.

Por tanto, el objetivo de este estudio es valorar la calidad percibida de los participantes en dos ediciones de una carrera popular de media distancia. 


\section{Método}

\subsection{Muestra}

La muestra estudiada estuvo conformada por un total de 708 participantes, 395 correspondientes a la I Edición de la prueba y 313 a la II Edición (Tabla 1). Respecto a la I Edición los participantes tenían una edad media de $40.42(+9.12)$, de los que $86.6 \%$ fueron hombres y solamente un $13.4 \%$ mujeres. Casi la mitad de los participantes tenían estudios universitarios (medios o superiores) con un 49.9\%, seguido de los que tenían Bachillerato y Formación Profesional con un 18.5\% y 18.0\% respectivamente. En cuanto a la situación laboral, la gran mayoría (85.6\%) disponía de una situación laboral activa, mientras que un $6.3 \%$ estaba desempleado/a y un $5.1 \%$ era estudiante.

Para la II Edición los hombres constituyeron el $77.00 \%$ del total de participantes, por el 23.0\% que supusieron las mujeres. El nivel de estudios que tuvieron fue de un 37.4\% estudios universitarios superiores, un 19.5\% Formación Profesional, Bachillerato (17.6\%), y un 17.3\% disponía de estudios universitarios medios. La situación laboral que tenían los participantes eran activos/as un 85.6\%, estudiantes un $6.4 \%$ y un $4.8 \%$ estaba desempleado/a.

\begin{tabular}{|c|c|c|c|c|}
\hline \multirow{2}{*}{ VARIABLE } & \multicolumn{2}{|c|}{ I EDICIÓN } & \multicolumn{2}{|c|}{ II EDICIÓN } \\
\hline & $\mathbf{N}$ & $\%$ & $\mathbf{N}$ & $\%$ \\
\hline \multicolumn{5}{|l|}{ Sexo } \\
\hline Hombres & 342 & 86.6 & 241 & 77.0 \\
\hline Mujeres & 53 & 13.4 & 72 & 23.0 \\
\hline \multicolumn{5}{|l|}{ Nivel de Estudios } \\
\hline Sin estudios & 1 & 0.3 & 0 & 0.0 \\
\hline Primaria & 12 & 3.0 & 11 & 3.5 \\
\hline Secundaria & 41 & 10.4 & 15 & 4.8 \\
\hline Bachillerato & 73 & 18.5 & 55 & 17.6 \\
\hline Formación Profesional & 71 & 18.0 & 61 & 19.5 \\
\hline Estudios Universitarios Medios & 83 & 21.0 & 54 & 17.3 \\
\hline Estudios Universitarios Superiores & 114 & 28.9 & 117 & 37.4 \\
\hline \multicolumn{5}{|l|}{ Situación Laboral } \\
\hline Activo/a & 338 & 85.6 & 268 & 85.6 \\
\hline Jubilado/a o Pensionista & 10 & 2.5 & 7 & 2.2 \\
\hline Desempleado/a & 25 & 6.3 & 15 & 4.8 \\
\hline Amo/a de casa & 2 & 0.5 & 3 & 1.0 \\
\hline Estudiante & 20 & 5.1 & 20 & 6.4 \\
\hline
\end{tabular}

Tabla 1. Descriptivos sociodemográficos 


\subsection{Instrumento}

El cuestionario utilizado sirve para valorar la calidad percibida en carreras populares de 22 ítems diseñado por Angosto (2014). Este instrumento está compuesto por un total de 4 dimensiones y 16 ítems de calidad atendiendo a los aspectos generales que debe disponer un evento como una carrera popular. La alternativa de respuesta es de tipo Likert de seis anclajes $(1-$ Muy en desacuerdo a 6 - Muy de acuerdo).

\subsubsection{Propiedades psicométricas}

Para comprobar la validez del cuestionario propuesto se aplicaron los tratamientos de fiabilidad y validez. La fiabilidad (cálculo de la consistencia interna de la escala), se determina a través del coeficiente alfa de Cronbach, obteniendo todos los factores índices adecuados en la mayoría de dimensiones (Tabla 2).

\begin{tabular}{|l|r|r|}
\hline ESCALA & Alfa de Cronbach & \multicolumn{1}{|c|}{ N $^{\mathbf{0}}$ Ítems } \\
\hline Comunicación & .758 & 3 \\
\hline Organización Logística & .805 & 4 \\
\hline Interacción Personal & .855 & 4 \\
\hline Servicios Complementarios & .708 & 5 \\
\hline Total Dimensiones & .914 & 16 \\
\hline
\end{tabular}

Tabla 2. Fiabilidad de factores y escala

\subsection{Procedimiento de administración}

La administración del cuestionario a los participantes en la carrera se realizó de forma telemática mediante la aplicación del cuestionario de forma online. Se puso en contacto con los organizadores de la prueba y la Federación de Atletismo de la Región de Murcia (FAMU) solicitando permiso para pasar el cuestionario con el fin de medir la calidad de la prueba.

Una vez obtenido el permiso se elaboró un mensaje de presentación del estudio para enviárselo junto con el enlace al cuestionario a los participantes que se distribuyó por medio de la FAMU mediante la lista de contactos de los participantes de la prueba. El mensaje se envió el día siguiente de la realización de la prueba, teniendo los participantes la información necesaria para rellenar el cuestionario de forma adecuada. 


\subsection{Análisis de datos}

El análisis de los datos se realizó con el paquete estadístico SPPS 19 con licencia de la Universidad de Murcia. Las pruebas que se hicieron fue análisis descriptivo y de frecuencias para la descripción de las variables, la prueba t de Student para corroborar la existencia de diferencias significativas entre las variables continuas y el Alfa de Cronbach para calcular la fiabilidad del instrumento y un Análisis Factorial Exploratorio (AFE) para comprobar la agrupación de los ítems.

\section{Resultados}

\subsection{Análisis Factorial Exploratorio}

Los resultados mostraron que el índice de Káiser - Meyer - Olkin (KMO) para la medida de adecuación muestral (Tabla 3), según Visauta, Martori y Cañas (2005), fue excelente. Según estos autores resultados por encima de 0.70 se consideran aceptables, presentando valores de 0.942 en este caso. En relación a los resultados del test de Esfericidad de Bartlett se obtuvieron resultados altamente significativos $(\mathrm{p}=.000)$ en la escala lo que permite realizar el análisis factorial exploratorio. El determinante de la matriz de correlaciones resulta bajo cerca de $0 \mathrm{y}$, por tanto, se considera adecuado.

\begin{tabular}{|c|r|r|r|}
\hline Escala & Determinante & KMO & \multicolumn{1}{|c|}{ Test de Esfericidad de Bartlett } \\
\hline \multirow{2}{*}{1} & \multirow{2}{*}{0.000} & 0.942 & $\mathrm{X}^{2} 3736.52$ \\
& & & gl 120 \\
\cline { 4 - 4 } & & Sig. 0.000 \\
\hline
\end{tabular}

Tabla 3. Criterios de pertinencia del análisis factorial escala de calidad percibida

Respecto a la varianza explicada (Tabla 4) los 16 ítems que conforman la escala de factores de calidad percibida explicaron un $65.4 \%$ de la varianza con una solución de 4 factores tal y como se observa en el gráfico de sedimentación (Figura 1). En el análisis factorial exploratorio (AFE), se observó que el $47.6 \%$ pertenece al primer factor, un $7.5 \%$ al segundo, un $5.5 \%$ al tercero y un $4.7 \%$ al cuarto. 


\begin{tabular}{|c|r|r|r|r|r|r|}
\hline & \multicolumn{4}{|c|}{ Autovalores iniciales } & \multicolumn{3}{c|}{ Suma datos al cuadrado de rotación } \\
\hline Factor & Total & \% Varianza & \% acumulado & \multicolumn{1}{c|}{ Total } & \% varianza & \% acumulado \\
\hline 1 & 7.62 & 47.6 & 47.6 & 3.5 & 22.0 & 22.0 \\
\hline 2 & 1.19 & 7.4 & 55.1 & 2.8 & 16.7 & 38.7 \\
\hline 3 & 0.88 & 5.5 & 60.6 & 2.2 & 13.7 & 52.5 \\
\hline 4 & 0.76 & 4.7 & 65.4 & 2.1 & 12.8 & 65.4 \\
\hline
\end{tabular}

Tabla 4. Varianza explicada del análisis factorial de la escala de calidad percibida

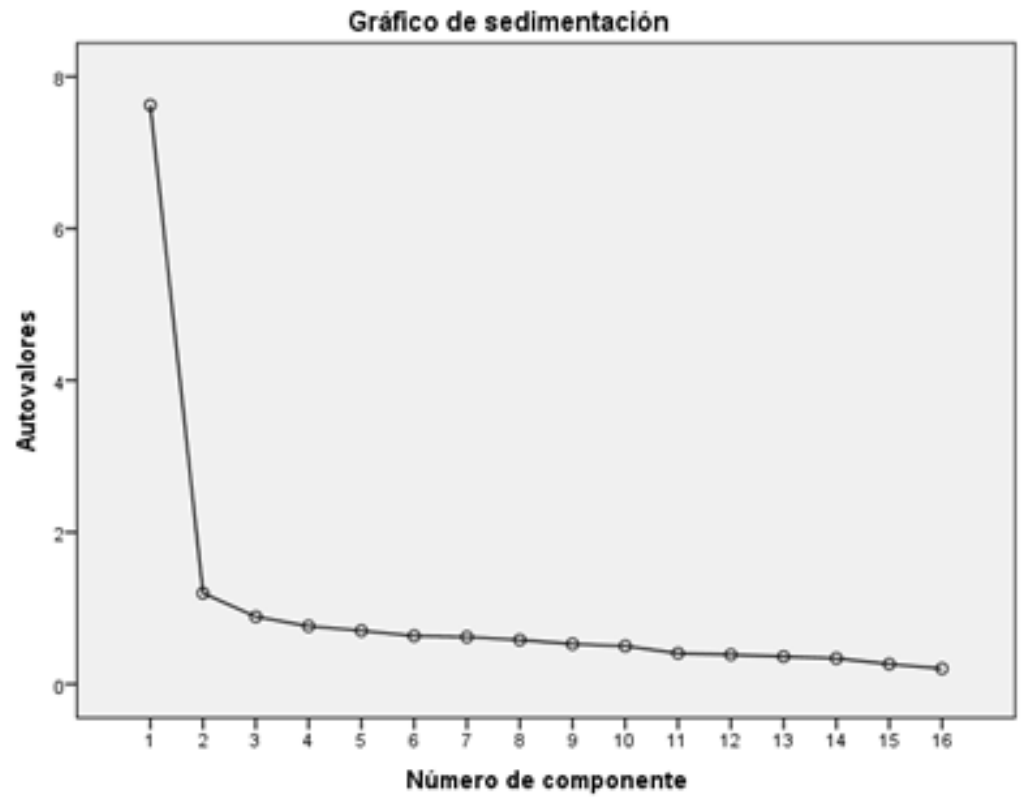

Figura 1. Gráfico de sedimentación escala de factores de calidad percibida

A continuación se presenta la matriz de componentes correspondiente a la escala de calidad percibida del evento (Tabla 5) que cuenta con una estructura interna formada por cuatro dimensiones, donde los pesos factoriales oscilan entre un mínimo de 0.361 del ítem 11 y un máximo de 0.846 del ítem 9, considerando que son representativos al presentar valores por encima de 0.30 . 


\begin{tabular}{|l|r|}
\hline \multicolumn{1}{|c|}{ Ítems } & \multicolumn{1}{|c|}{ Cargas } \\
\hline Factor 1: Interacción personal & 0.725 \\
\hline Ítem 4. He recogido fácilmente el dorsal sin realizar mucha espera. & 0.580 \\
\hline Ítem 5. La organización cumple con los horarios previstos. & 0.746 \\
\hline Ítem 6. El personal de la organización está dispuesto a ayudar/dar consejos. & 0.785 \\
\hline Ítem 7. Los voluntarios atienden con amabilidad. & 0.701 \\
\hline Factor 2: Comunicación & 0.645 \\
\hline $\begin{array}{l}\text { Ítem 1. La carrera cuenta con una buena promoción y difusión, aportando suficiente información práctica sobre } \\
\text { la misma. }\end{array}$ & 0.572 \\
\hline Ítem 2. Durante el evento se da información clara y precisa para conocer el desarrollo de la competición. & 0.371 \\
\hline Ítem 3. Ha sido sencillo realizar la inscripción. & 0.735 \\
\hline Factor 3: Servicios Complementarios & 0.476 \\
\hline Ítem 12. Los resultados y el podium son visibles por todos los espectadores. \\
\hline Ítem 13. El recorrido de la carrera cuenta con los suficientes puntos de avituallamiento y son adecuados. \\
\hline Ítem 14. La bolsa del corredor es adecuada y completa. & .541 \\
\hline $\begin{array}{l}\text { Ítem 15. La carrera cuenta con servicios de apoyo suficientes (baños, vestuarios, guardarropa, zonas de masajes, } \\
\text { stands, etc.). }\end{array}$ & 0.644 \\
\hline Ítem 16. Cerca de la salida/meta de la carrera existen lugares de fácil accesibilidad comercial (cafeterías, bares,...). & \\
\hline Factor 4: Infraestructura logística & 0.763 \\
\hline Ítem 8. La señalización del evento permite llegar fácilmente a su lugar de inicio. & 0.846 \\
\hline Ítem 9. Existe aparcamiento suficiente cerca de la zona de inicio o de llegada de la carrera. \\
\hline $\begin{array}{l}\text { Ítem 10. Los elementos materiales que utiliza el evento son visualmente atractivos (pancartas, vallas, salida, meta, } \\
\text { recorrido...). }\end{array}$ & 0.454 \\
\hline Ítem 11. El recorrido de la carrera está bien señalizado y es seguro. & 0.361 \\
\hline
\end{tabular}

Tabla 5. Estructura factorial escala de calidad percibida

Por último, para concluir el análisis factorial exploratorio, en la Tabla 6 se expone la estructura final de la herramienta después de realizar este análisis. El primer factor estuvo compuesto por un total de cuatro ítems, al igual que el factor 4 de infraestructura logística. El factor 2 de comunicación estuvo compuesto por tres ítems, mientras que los servicios complementarios con cinco ítems.

\begin{tabular}{|c|c|c|}
\hline Factor & Denominación & Ítems \\
\hline 1 & Dorsal, horarios, personal, voluntarios, & $4,5,6,7$ \\
\hline 2 & Promoción, información, inscripción, & $1,2,3$ \\
\hline 3 & $\begin{array}{c}\text { Resultados, avituallamiento, bolsa, servicio } \\
\text { apoyo, locales comerciales }\end{array}$ & $12,13,14,15,16$ \\
\hline 4 & $\begin{array}{c}\text { Señalización, aparcamiento, elementos } \\
\text { materiales, recorrido }\end{array}$ & $8,9,10,11$ \\
\hline
\end{tabular}

Tabla 6. Estructura final de la escala de calidad percibida 


\subsection{Análisis descriptivo factores calidad percibida en general}

Los resultados relativos a lo descriptivos generales de los factores de calidad atendiendo a la edición de la prueba se indican en la Tabla 7.

En los resultados relativos a la I Edición arrojaron valoraciones muy altas, todas ellas por encima de 5 puntos. La dimensión mejor valorada fue la interacción personal con un valor de 5.80 sobre los 6 puntos posibles. En segundo lugar, la infraestructura logística fue la dimensión más valorada y similares a los servicios complementarios con un 5.45 y 5.41 respectivamente. La comunicación, a pesar de ser el factor peor valorado obtuvo una puntuación satisfactoria de 5.21 .

En cuanto a los resultados de la II Edición, el factor humano vuelve a ser el más valorado con 5.11, volviendo a poner en valor al personal de la organización. El resto de dimensiones mostraron resultados ligeramente inferiores con puntuaciones próximas a 5, la comunicación fue el segundo factor más valorado con 4.83 puntos, seguido de los servicios complementarios (4.79) e infraestructura logística (4.70).

En general, los resultados obtenidos en la I Edición de la prueba fueron superiores a los de la II Edición en todas las dimensiones, presentando una mayor variabilidad en la respuesta de los participantes de la II Edición. Existieron diferencias significativas en todas las dimensiones atendiendo a la prueba con valoras de $\mathrm{p}<.000$ en todas ellas.

\begin{tabular}{|c|r|r|r|}
\hline \multirow{2}{*}{ Dimensión } & \multicolumn{2}{|c|}{ General } & \multirow{2}{*}{ Sig. } \\
\cline { 2 - 4 } & \multicolumn{1}{|c|}{ M } & \multicolumn{1}{|c|}{ DT } & \\
\hline Comunicación & & & .000 \\
\hline I Edición & 5.21 & .56 & \\
\hline II Edición & 4.83 & .89 & \\
\hline Interacción Personal & & & .000 \\
\hline I Edición & 5.80 & .36 & \\
\hline II Edición & 5.11 & .92 & \\
\hline Infraestructura Logística & & & .000 \\
\hline I Edición & 5.45 & .55 & \\
\hline II Edición & 4.70 & .89 & \\
\hline Servicios Complementarios & & & .000 \\
\hline I Edición & 5.41 & .72 & \\
\hline II Edición & 4.79 & 1.01 & \\
\hline
\end{tabular}

Tabla 7. Descriptivo de factores calidad percibida en general 


\subsection{Análisis descriptivo factores calidad percibida según el sexo de los participantes}

Los resultados de las dimensiones de calidad respecto al sexo de los participantes en ambas ediciones se muestran en la Tabla 8.

Respecto a los hombres se puede observar que los resultados de la I Edición son superiores a lo obtenidos en la segunda prueba. La dimensión más valorada fue la de interacción personal con unos valores excelentes en la I Edición de 5.79 y muy buenos en la segunda (5.09). La infraestructura logística para la I Edición supuso el segundo factor mejor evaluado con una media de 5.44. sin embargo para la segunda edición fue el peor valorado de todos (4.69 puntos).

Las otras dimensiones los hombres valoraron en tercer lugar, para la I Edición los servicio complementarios (5.40) y, por último, la comunicación (5.19). En cambio, la comunicación fue el segundo factor con mayor puntuación (4.80) en la segunda edición de la prueba y los servicios complementarios la tercera con un valor de 4.76 .

\begin{tabular}{|c|c|c|c|c|c|}
\hline \multirow{2}{*}{ Dimensión } & \multicolumn{2}{|c|}{ Hombre } & \multicolumn{2}{|c|}{ Mujer } & \multirow{2}{*}{ Sig. } \\
\hline & M & DT & M & DT & \\
\hline \multicolumn{6}{|l|}{ Comunicación } \\
\hline I Edición & 5.19 & .56 & 5.31 & .58 & .189 \\
\hline II Edición & 4.80 & .86 & 4.95 & .98 & .217 \\
\hline \multicolumn{6}{|l|}{ Interacción Personal } \\
\hline I Edición & 5.79 & .37 & 5.88 & .32 & .100 \\
\hline II Edición & 5.09 & .90 & 5.19 & .97 & .391 \\
\hline \multicolumn{6}{|l|}{ Infraestructura Logística } \\
\hline I Edición & 5.44 & .56 & 5.50 & .49 & .465 \\
\hline II Edición & 4.69 & .86 & 4.74 & 1.00 & .736 \\
\hline \multicolumn{6}{|c|}{ Servicios Complementarios } \\
\hline I Edición & 5.40 & .73 & 5.47 & .65 & .500 \\
\hline II Edición & 4.76 & .98 & 4.87 & 1.11 & .419 \\
\hline
\end{tabular}

Tabla 8. Descriptivo de factores calidad percibida según el sexo

En cuanto a las mujeres. al igual que los hombres, tuvieron una mejor valoración de la I Edición respecto de la II Edición. La dimensión personal, al igual que la percepción general y de los hombres, fue el más valorado con una puntuación prácticamente perfecta en la primera edición de 5.88 puntos sobre 6 posibles y una muy buena valoración de 5.19 puntos en la segunda prueba.

Para la I Edición el segundo factor más valorado fue la infraestructura logística, seguido muy de cerca por los servicios complementarios con unos valores de 5.50 y 5.47 respectivamente. Por el contrario, en 
la II Edición la dimensión de comunicación tuvo una valoración de 4.95 puntos, los servicios complementarios 4.87 y, en último lugar, la infraestructura logística con una puntuación de 4.74, todas ellas buenas aunque más bajas respecto a la edición anterior.

No se observaron diferencias significativas entre las valoraciones de hombres y mujeres en cada una de las dimensiones para las dos ediciones de la prueba. Sin embargo, sí existieron diferencias significativas entre la opinión de los hombres y las mujeres entre la primera y la segunda edición de la carrera popular, con niveles de $\mathrm{p}<.000$ en todas ellas, salvo la opinión de mujeres para la dimensión de servicios complementarios en mujeres que obtuvo un nivel de significación de $\mathrm{p}=.021$.

\section{Discusión}

Conocer la opinión de los participantes de una carrera popular puede ayudar considerablemente a los organizadores del evento deportivo a poder elaborar unas estrategias más adecuadas y eficaces en futuras ediciones poder mejorar los aspectos que, bajo la percepción de las personas que han vivido la prueba, no han presentado la calidad suficiente para cumplir las expectativas que tenían generadas con antelación al día de la carrera.

La segmentación de la información en la valoración de la calidad percibida atendiendo a los diferentes datos sociodemográficos que se tengan va a resultar de ayuda a los gestores y empresas deportivas para la mejora de los servicios con dicha información (Calabuig, Burillo, Crespo, Mundina \& Gallardo, 2010), al disponer de una información más detallada pudiendo concretar más la atención en un perfil concreto de usuario al que enfocar el evento deportivo.

Atendiendo a los resultados generales obtenidos en esta investigación y en comparación con otros estudios previos se puede determinar que la dimensión comunicación se obtuvieron valores similares, aunque ligeramente inferiores, en la primera edición a los obtenidos por Angosto, Ibáñez-Pérez y Morales-Baños (2014) sobre una media maratón. En ambas ediciones se obtuvo una mejor valoración de esta dimensión en la valoración de la calidad percibida en una prueba de triatlón realizado por Angosto, Morán-Navarro, Martínez-Cava y López-Gullón (2016).

Respecto a la dimensión de interacción personal, se trata de uno de los aspectos más estudiados dentro de la literatura internacional, al considerarse el factor humano como el elemento más importante dentro de una organización deportiva para la realización eficiente de los servicios (Bodet, 2006). Este factor 
tuvo una alta valoración en ambos pruebas, al igual que sucedió en los en los trabajos sobre la valoración de la calidad percibida de Alexandris, Kouthouris y Meligdis (2006) en esquiadores de un resort, Angosto et al. (2014) en corredores de media maratón, Calabuig et al. (2010) sobre espectadores de atletismo, Crespo (2011) en espectadores de baloncesto, y Pérez-Campos (2010) en espectadores del campeonato de España y del Mundo de Atletismo en pista cubierta.

Otros estudios utilizaron el modelo SERVQUAL de Parasuraman, Zeithaml y Berry (1988) sobre eventos deportivos como es el caso Mundina y Calabuig (2011) sobre espectadores y deportistas de los Juegos del Mediterráneo de Almería, Pérez-Campos (2010) en deportistas participantes en el Campeonato de España y del Mundo de Atletismo en pista cubierta o Theodorakis, Kambitsis, Laios y Koustelios, (2001) en espectadores de baloncesto. En estos trabajos se valoraron positivamente aspectos como la fiabilidad, la capacidad de respuesta y la empatía.

La dimensión organización logística obtuvo resultados inferiores a los obtenidos por Angosto et al. (2016) sobre la valoración de una prueba de triatlón, donde la organización logística fue el factor más valorado por los participantes, donde este aspecto siempre es mucho más completo en este deporte donde se conjuntan tres disciplinas deportivas como son el nado, el ciclismo y la carrera con muchas características particulares para cada segmento. Por el contrario, en estas pruebas estuvo mejor valoraras que en el trabajo de Angosto (2014) sobre los participantes en una media maratón.

En relación al último factor de servicios complementarios la I Edición obtuvo mejores resultados que los obtenidos por Angosto et al. $(2014,2016)$ donde fueron de las dimensiones peor valoraras y Crespo (2011) con una valoración de 5.40 en este caso sobre 7 puntos y no 6 como en las anteriores. La II Edición estuvo peor valoración que la que tuvieron estos autores en sus respectivas investigaciones.

Por último, las valoraciones de las diferentes dimensiones de calidad en función del sexo de los participantes puede decirse que los resultados de este estudio coinciden con los obtenidos por Calabuig et al. (2010) donde las mujeres tuvieron una mejor opinión del evento deportivo al presentar unas puntuaciones más altas que los hombres, al igual que el factor personal que en el trabajo de Pérez-Campos (2010) tanto en los espectadores como los deportistas. Este hecho pone de manifiesto que las mujeres tienen una opinión más crítica que los hombres a pesar de estar menos presentes en la participación de eventos deportivos, ya sea como espectador o deportista. 


\section{Conclusiones}

Las principales conclusiones que se obtienen de este estudio tras el análisis de los resultados son las siguientes:

- La I Edición de la prueba tuvo una mejor valoración de la calidad percibida que la II Edición, encontrándose diferencias significativas en todas las dimensiones, produciéndose, por tanto, un descenso del nivel de calidad de la prueba.

- La dimensión de interacción personal ha sido el factor mejor evaluado de todos en ambas ediciones, tanto de forma general como para hombres y mujeres.

- La valoración de la calidad percibida que tuvieron las mujeres ha sido superior en todas las dimensiones y en ambas ediciones, aunque no se encontraron diferencias significativas entre sexos en ningún caso.

\section{Aplicación práctica}

Los investigadores de este estudio consideran que el empleo de este tipo de instrumento aporta numerosas oportunidades para el análisis de la calidad percibida en los participantes de eventos deportivos en concreto de las carreras populares. Este tipo de pruebas deportivas son tan comunes hoy en día que se celebran varias de ellas cada mes, por lo que se hace necesario poder medir cada prueba para que los participantes y organizadores puedan discriminar cuales pueden ser las mejores a la hora de elegir a qué pruebas asistir. Los resultados que se obtengan con su aplicación puede ser de gran utilidad para las organizaciones y empresas deportivas, tanto públicas como privadas, que estén destinadas o se dediquen a la organización eventos deportivos para tratar de hacerlo más atractivo y atraer a un mayor número de participantes.

\section{Referencias}

Afthinos, Y., Theodorakis, N.D., \& Nassis, P. (2005). Customers' expectations of service in greek fitness centers. Gender, age, type of sport center, and motivation differences. Managing Service Quality, 15(3), 245-258. http://dx.doi.org/10.1108/09604520510597809 
Alexandris, K., Kouthouris, C., \& Meligdis, A. (2006). Increasing customers' loyalty in a skiing resort: The contribution of place attachment and service quality. International Journal of Contemporary Hospitality Management, 18(5), 414-425. http://dx.doi.org/10.1108/09596110610673547

Angosto, S., Ibáñez-Pérez, R., \& Morales-Baños, V. (2014). Valoración de la calidad percibida, valor percibido y satisfacción de los participantes en una carrera popular de media distancia. En M.A. Espitia, L.I. García-Cebrián, A. Muñoz-Pocar, R. Serrano-Lázaro, \& J. Gutiérrez-Ilarduya (Eds.), V Congreso Iberoamericano de Economía del Deporte. El deporte: Dinamizador económico (pp. 23-26). Zaragoza, España.

Angosto, S., Morán-Navarro, R., Martínez-Cava, A., \& López-Gullón, J.M. (2016). Calidad percibida en una prueba de triatlón. SporTK: Revista Euroamericana de Ciencias del Deporte, 5(1), 81-84.

Angosto, S.S. (2014). Diseño y validación de un cuestionario sobre calidad percibida en eventos deportivos populares (CAPPEP). Trabajo de Fin de Máster. Murcia: Universidad de Murcia.

Bodet, G. (2006). Investigating customer satisfaction in a health club context by an application of the tetraclasse model. European Sport Management Quarterly, 6(2), 149-165. http://dx.doi.org/10.1080/16184740600954148

Calabuig, F., Burillo, P., Crespo, J., Mundina, J.J., \& Gallardo, L. (2010). Satisfacción, calidad y valor percibido en espectadores de atletismo. Revista Internacional de Medicina y Ciencias de la Actividad Física y el Deporte, 10(40), 577-593. Disponible online en: http://cdeporte.rediris.es/revista/revista40/artsatisfaccion182.htm

Calabuig, F., Molina, N., \& Núñez, J. (2012). Una aplicación inicial del modelo tridimensional de calidad de servicio en centros deportivos privados. E-Balonmano: Revista de Ciencias del Deporte, 8(1), 67-81.

Chelladurai, P., \& Chang, K. (2000). Targets and standards of quality in sport services. Sport Management Review, 3(1), 1-22. http://dx.doi.org/10.1016/S1441-3523(00)70077-5

Clemes, M., Brush, G., \& Collins, M. (2011). Analysing the professional sport experience: A hierchical approach. Sport Management Review, 14, 370-388. http://dx.doi.org/10.1016/j.smr.2010.12.004

Crespo, J. (2011). Los eventos deportivos: Calidad de servicio, valor percibido y satisfacción de los espectadores. Valencia: Universidad de Valencia.

Gálvez, P., Morales-Sánchez, V., Hernández-Mendo, A., Pérez-López, R., \& López-Linares, A. (2012). Evaluación en gestión deportiva: Calidad de servicios. Revista de Ciencias del Ejercicio FOD, 7(7), 29-37. 
Grönroos, C. (1984). A service quality model and its marketing implications. European Journal of Marketing, 18(4), 36-44. http://dx.doi.org/10.1108/EUM0000000004784

Mármol, A., Orquín Castrillón, F.J., \& Sainz de Baranda, P. (2010). La infraestructura y el equipamiento, la prescripción del ejercicio y los servicios ofertados como índices de calidad de los centros fitness de Murcia. [Versión electrónica]. Cuadernos de Psicología del Deporte, 10(2), 85-91.

Mundina, J., \& Calabuig, F. (2011). La Calidad de servicio percibida de un gran evento deportivo como indicador de gestión. Journal of Sports Economics y Management, 1(1), 31-47.

Parasuraman, A., Zeithaml, V., \& Berry, L. (1988). SERVQUAL: A multiple-item scale for measuring consumer perceptions of service quality. Journal of Retailing, 64(1), 12-40.

Parasuraman, A., Zeithaml, V., \& Berry, L. (1985). A conceptual model of service quality and its implications for future research. Journal of Marketing, 49, 41-50. http://dx.doi.org/10.2307/1251430

Pérez-Campos, C. (2010). Análisis de la calidad del servicio en los eventos deportivos. Calidad percibida y satisfacción de los espectadores y de los deportistas. Valencia: Universidad de Valencia.

Rosa, M., Castellanos, M., \& Palacios, B. (2012). Calidad percibida por el espectador de fútbol. Revista de psicología del deporte, 21(1), 25-33.

Shone, A., \& Bryan, P. (2010). Successful event management: A practical handbook. London: CENGAGE Learning.

Theodorakis, N., Kambitsis, C., Laios, A., \& Koustelios, A. (2001). Relationship between measures of service quality and satisfaction of spectators in professional sports. Managing Service Quality, 11(6), 431-438. http://dx.doi.org/10.1108/09604520110410638

Visauta, B., Martori, I., \& Cañas, J.C. (2005). Análisis estadístico con SPSS para Windows. España: McGrawHill. 